Erratum

\title{
Erratum to "The Comparative Study of the Effectiveness of Cimetidine, Ranitidine, Famotidine, and Omeprazole in Treatment of Children with Dyspepsia"
}

\author{
Seyed Mohsen Dehghani, Mohammad Hadi Imanieh, \\ Roya Oboodi, and Mahmood Haghighat
}

Gastroenterohepatology Research Center, Pediatric Gastroenterology Department, Nemazee Hospital, Shiraz University of Medical Sciences, Shiraz 71937-11351, Iran

Correspondence should be addressed to Seyed Mohsen Dehghani; dehghanism@sums.ac.ir

Received 19 February 2013; Accepted 10 March 2013

Copyright (c) 2013 Seyed Mohsen Dehghani et al. This is an open access article distributed under the Creative Commons Attribution License, which permits unrestricted use, distribution, and reproduction in any medium, provided the original work is properly cited.

In the paper "The comparative study of the effectiveness of Cimetidine, Ranitidine, Famotidine, and Omeprazole in treatment of children with dyspepsia" by S. M. Dehghani et al., published in ISRN Pediatrics, Volume 2011, Article ID 219287, one sentence needs to be amended as it is incorrect or incomplete. In the abstract, in line 9, the correct sentence should read " $21.6 \%$ for Cimetidine, $43.2 \%$ for Ranitidine, 44.4\% for Famotidine, and 53.8\% for Omeprazole ( $P=$ .024)." 


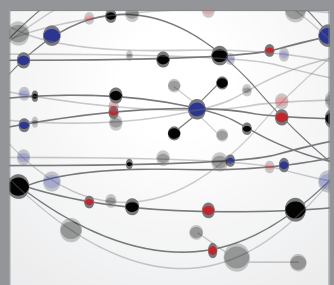

The Scientific World Journal
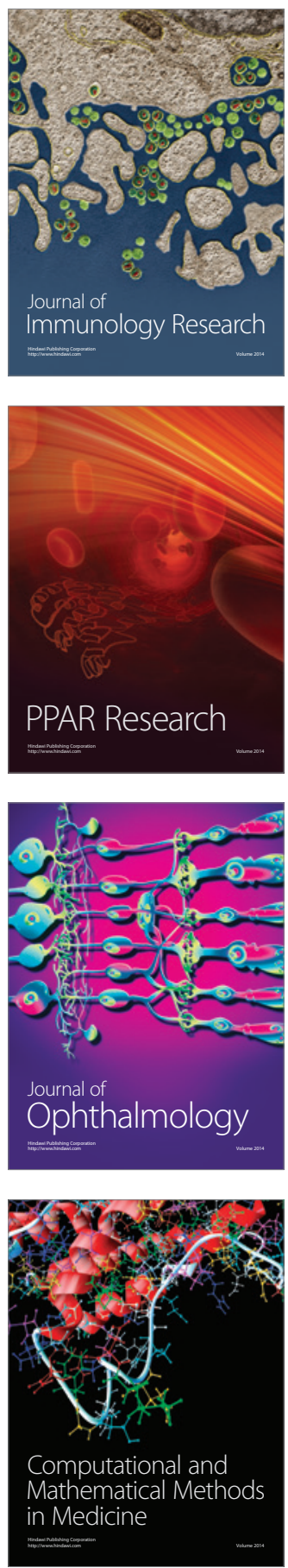

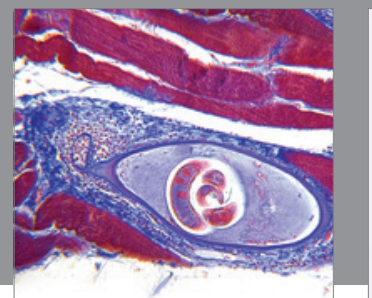

Gastroenterology

Research and Practice
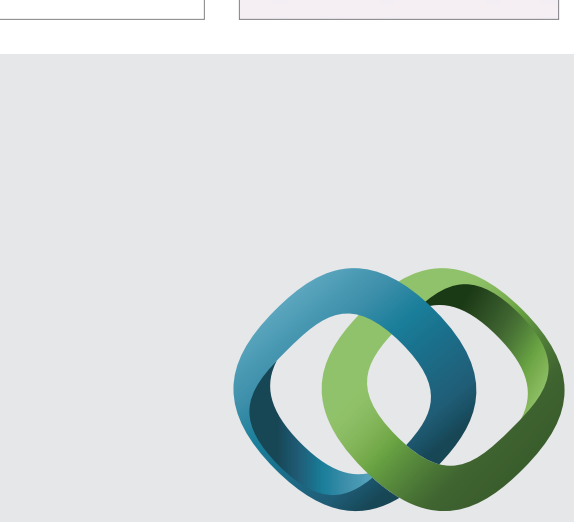

\section{Hindawi}

Submit your manuscripts at

http://www.hindawi.com
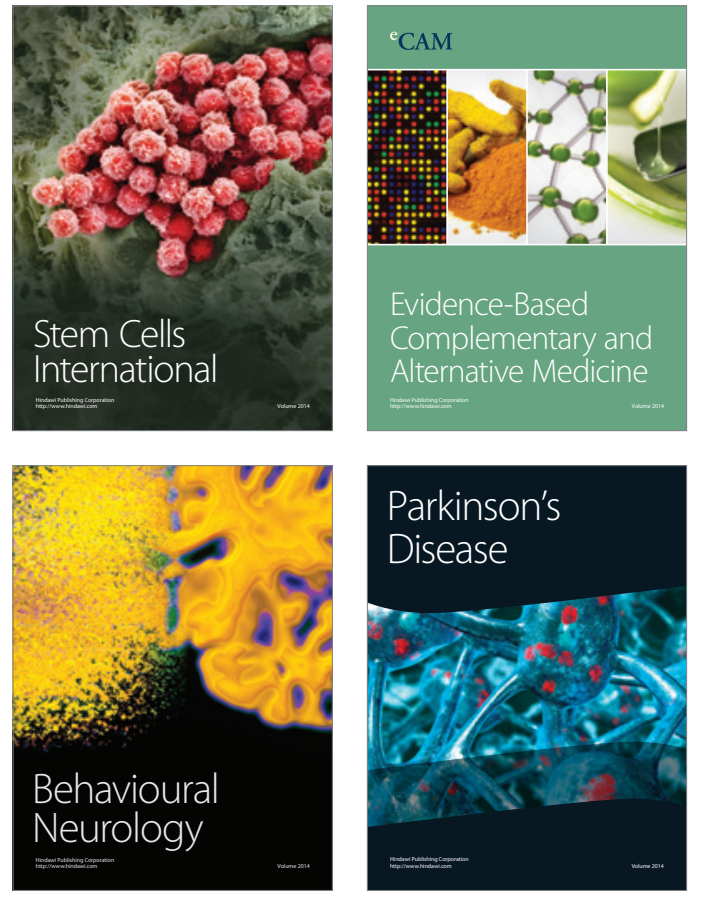
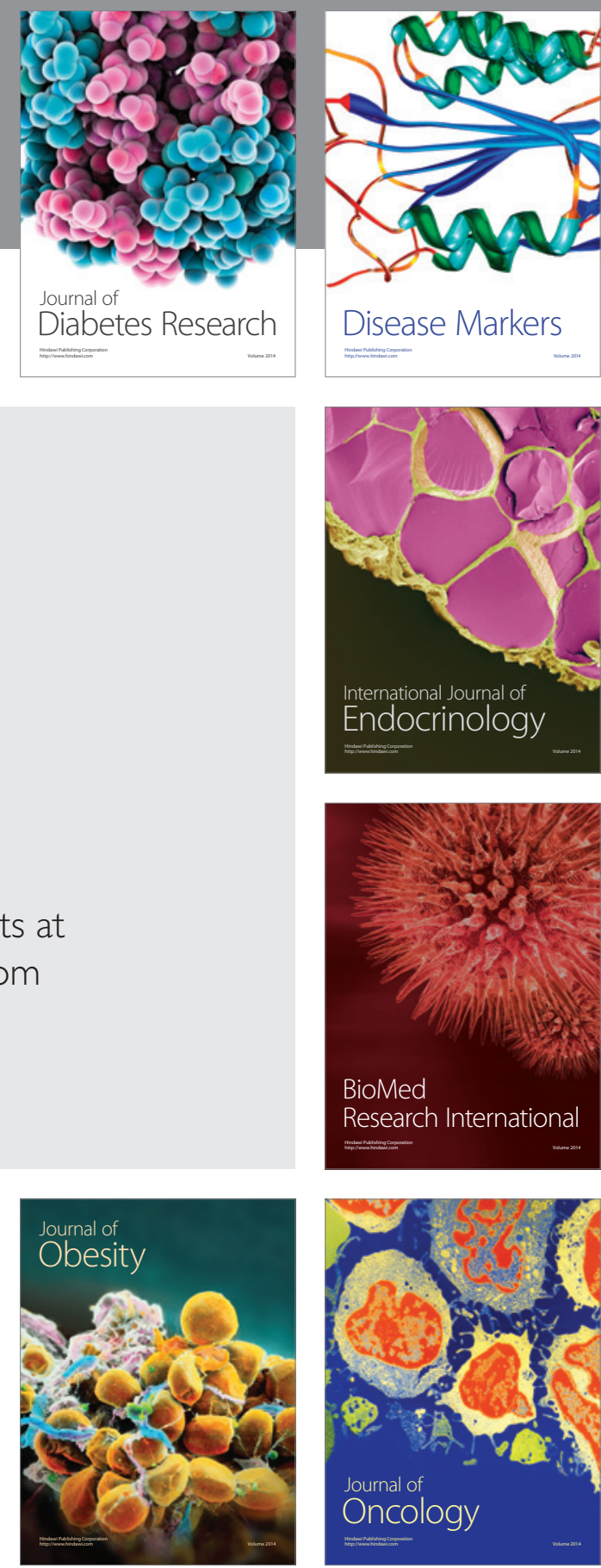

Disease Markers
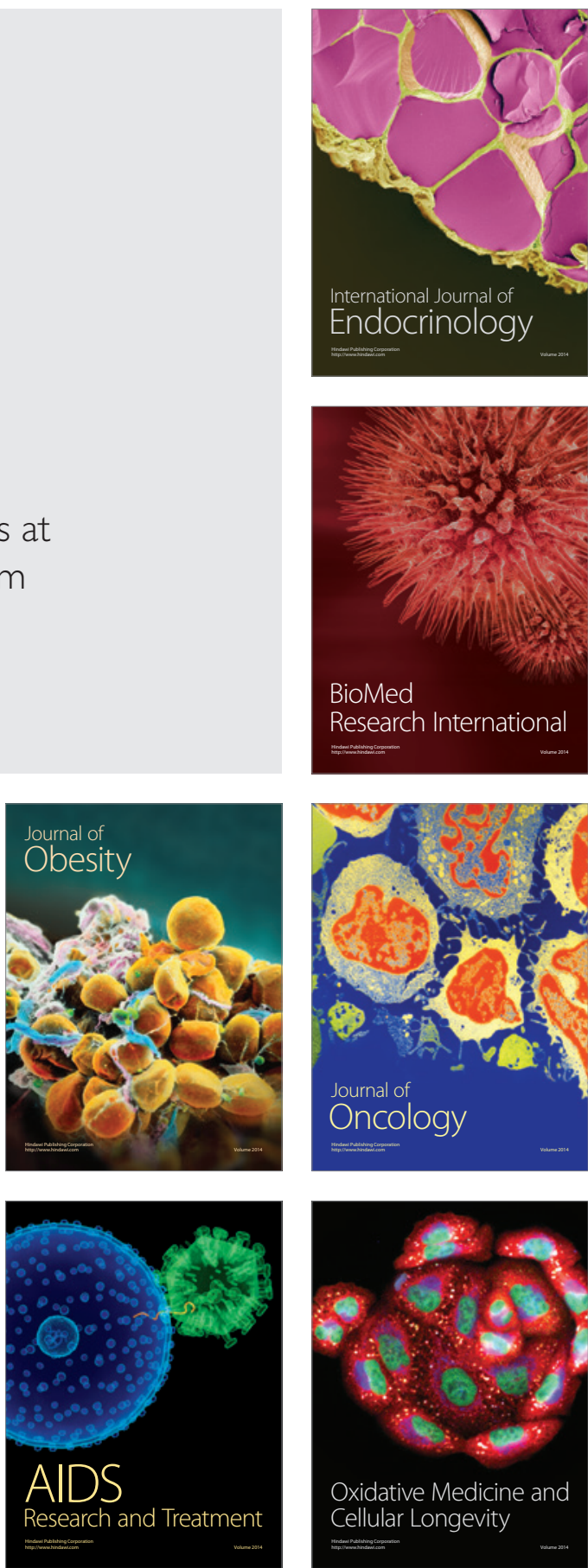